\title{
Figures
}

I.1 'Enterrement du dernier omnibus'. Agence Rol, 11 January 1913. Bibliothèque nationale de France

I.2 'Ordonnance sur l'établissement des voitures publiques dites omnibus'. 1828. Bibliothèque historique de la ville de Paris

I.3 Charles-Valentin Alkan, 'Les Omnibus', variation for piano in $\mathrm{C}$ major. 1828. Frontispiece. Author's copy

I.4 Tableau synoptique de la circulation des omnibus à correspondances de Paris. 1840. Collection RATP

I.5 Plan de la ville de Paris représentant les nouvelles voitures publiques à 30 centimes la course. 1829. Bibliothèque historique de la ville de Paris

I.6 Honoré Daumier, 'Le mauvais côté des nouveaux omnibus'. Le Charivari, 4 September 1856. Courtesy of the Robert D. Farber University Archives \& Special Collections Department, Brandeis University

I.7 Honoré Daumier, 'Quinze centimes un bain complet. . parole, c'est pas payé!...'. Le Charivari, 30 August 1856. Courtesy of the Robert D. Farber University Archives \& Special Collections Department, Brandeis University

I.8 Honoré Daumier, 'Commençant à trouver que l'impériale des omnibus n'est pas une invention aussi agréable qu'il se l'était d'abord imaginé'. Le Charivari, 10 February 1858. Corcoran Collection, National Gallery of Art, Washington, DC

I.9 Cham, 'Les nouveaux omnibus du boulevard pendant l'hiver'. 1840

I.10 'L'enterrement de "la dernière" omnibus'. Excelsior, 12 January 1913. Bibliothèque nationale de France

I.11 'Feu l'Omnibus enterré joyeusement'. La Presse, 12 January 191324

I.12 Charles Vernier. 'Entrée dans un omnibus, rue Notre-Dame de Lorette'. La Crinolonomanie. 1856. Bibliothèque nationale de France 


\section{Engine of modernity}

1.1 M. Sahib, 'L'intérieur d'un omnibus'. 1874. Bibliothèque historique de la ville de Paris

1.2 'Les cochers de fiacres aux prises avec ceux des omnibus'. c. 1830. Musée Carnavalet/Roger-Viollet

1.3 Jean Georges Frey, 'Les Dames Blanches et le fiacre: Y-s-auront le tems d'engraisser nos chevaux!!!!!. 1828. Musée Carnavalet/Roger-Viollet

2.1 Honoré Daumier, '-N'est-il pas vrai, brave turco, que vous préférez les Françaises aux Africaines? -Chut! .. . ma bonne... tu vois bien que tu vas le faire rougir!'. Le Charivari, 31 August 1859. Courtesy of the Robert D. Farber University Archives \& Special Collections Department, Brandeis University

2.2 M. de Penne, 'Le public des omnibus dans les bureaux de correspondence'. L'Illustration, 31 July 1868

2.3 Honoré Daumier, En omnibus. 1864. Daumier-Register

2.4 Honoré Daumier, 'Une rencontre désagréable'.

La Comédie humaine, 1843. Courtesy of the Robert D. Farber University Archives \& Special Collections Department, Brandeis University

2.5 'Un mariage en omnibus'. 1882. Cover, sheet-music score. Author's photo

2.6 'Mon voisin d'omnibus'. 1888. Cover, sheet-music score. Author's photo

3.1 A. Darjou, 'Actualités. - Oui Messieurs c'est quarte sous l'impériale; pour trois sous vous n'avez plus que le droit de suivre en courant'. n.d. Bibliothèque historique de la ville de Paris

3.2 Honoré Daumier, 'Désolé, citoyenne, mais on ne reçoit pas de chien. -Aristocrate, va!. 1848. Courtesy of the Robert D. Farber University Archives \& Special Collections Department, Brandeis University

3.3 Gobert, 'Intérieur d'un équipage de la petite propriété. 1829. Bibliothèque historique de la ville de Paris

3.4 Honoré Daumier, 'Madeleine-Bastille. Un zeste, un rien. . . et lomnibus se trouve complet'. 1862. Courtesy of the Robert D. Farber University Archives \& Special Collections Department, Brandeis University

3.5 Honoré Daumier, 'Intérieur d'un omnibus. Entre un homme ivre et un charcutier'. 1839. Courtesy of the Robert D. Farber University Archives \& Special Collections Department, Brandeis University 


\section{Figures}

4.1 Emile Dartès, Contes en omnibus. Illustration, 'Madeleine-Bastille'. 1894. Author's photo

4.2 Emile Dartès, Contes en omnibus. Illustration, 'Madeleine-Bastille'. 1894. Author's photo

4.3 'Madame Crinoliska faisant son entrée dans un omnibus'. Paris Grotesque, 1859. Musée Carnavalet/Roger-Viollet

4.4 'Une poule mouillée'. 1865. Based on a painting by Morlon.

Collection RATP 